\title{
A numerical method to compute Euler potentials for non dipolar magnetic fields
}

\author{
C. Peymirat, D. Fontaine \\ CETP/UVSQ, 10-12 Avenue de l'Europe, 78140 Velizy, France
}

Received: 16 December 1997 / Revised: 19 August 1998 / Accepted: 3 September 1998

\begin{abstract}
The magnetospheric magnetic field may be conveniently described by two scalar functions $(\alpha, \beta)$, known as the Euler potentials. They are not uniquely defined, and they may be difficult to derive for configuration more complex than a simple dipole. We propose here a simple numerical method to compute one possible pair $(\alpha, \beta)$. In magnetospheric regions of closed field lines, $\alpha$ can be chosen as a function of the tube volume of unit magnetic flux. The method can be applied to a wide class of magnetic fields which describe the magnetospheric domain of closed field lines and the conjugated ionosphere. Here, it is used with the T87 Tsyganenko model. The results coincide with the dipolar potentials at close distances from the Earth. At larger distances, they display an increasing distortion with the radial distance (or the invariant latitude in the ionosphere) and the magnetic activity. In the magnetosphere, the contours of $\alpha$ and $\beta$ are stretched towards the nightside. In the ionosphere, they also extend towards the nightside and present major distortions in a narrow ring at the polar cap boundary, which maps distant boundary layers in the magnetosphere.
\end{abstract}

Key words. Ionosphere (ionosphere-magnetosphere interactions; modeling and forecasting). Magnetospheric physics (plasma convection).

\section{Introduction}

The large-scale convection of the magnetospheric plasma is strongly related to the topology of the magnetic field $\mathbf{B}$ and the coupling with the ionosphere. The curvature and the gradients of the magnetic field control the drift of the magnetospheric plasma. Particle ex-

Correspondence to: C. Peymirat change, current circulation and electric field transmission between the magnetosphere and the ionosphere contribute to couple the magnetospheric motion strongly to the ionospheric motion. A convenient way to solve the magnetospheric transport is the use of the Euler potentials $(\alpha, \beta)$ as system coordinates. They are defined as:

$\mathbf{B}=\nabla \alpha \times \nabla \beta$

The interest of this representation has been extensively discussed by Stern (1967, 1970, 1976, 1994b). Briefly, it includes directly the expression of the magnetic field lines: $\alpha=$ constant and $\beta=$ constant. Two points belong to the same field line only if they have the same Euler potentials, at least for a field line topology sufficiently regular as is the case for the planetary fields (Stern, 1994b). This property reveals itself to be very powerful as a means of studying the transport of the magnetospheric flux tubes, and their coupling with the ionospheric plasma: the formulation of the basic transport equations is much simplified by choosing the Euler potentials as a reference frame (Böstrom, 1975; Peymirat and Fontaine, 1994b; Stern, $1994 \mathrm{~b}$ and references therein). This representation has been widely used in the inner magnetosphere where the magnetic field is essentially dipolar and simple analytical expressions can be derived for the Euler potentials (Stern, 1976 and references therein). This method can be applied to outer regions of the magnetosphere, or to dynamical situations like substorms, where the magnetic field departs significantly from the dipole (see Hilmer and Voigt, 1995; Tsyganenko, 1996; Ostapenko and Maltsev, 1997; Tsyganenko, 1997 for the most recent magnetic field models).

The derivation of the Euler potentials is very easy for a dipolar magnetic field but is rather difficult for any type of magnetic field. Equation (1) is a highly non linear differential equation, and from a practical point of view, it restricts the use of Euler potentials. For current-free fields, they can be exactly derived for a limited class of magnetic fields (Stern, 1994a) or approximately obtained with perturbations methods (Stern, 1967). Stern (1987) 
showed that the stretching of magnetic field lines in the tail produces realistic models but the Euler potentials are not in general explicitly known. Alternatively, they can be numerically computed. Cheng (1995 and references therein) solved the Grad-Shafranov equation relating the Euler potentials to the pressure of the magnetospheric plasma. Euler potentials were computed from a given pressure profile. Tsyganenko and Stern (1996) and Khurana (1997) modelled the Euler potentials as theoretical functions fitted to databases, to reproduce respectively the distribution of the field aligned currents and the jovian magnetic field. More recently, Ho et al. (1997) developed a general numerical method to compute the Euler potentials associated with the Neptune's magnetic field. This method uses the relationship between Euler potentials and the magnetic flux per unit area. The Euler potentials are similar to those of a dipole in the regions where the magnetic field is dipole-like.

The Euler potentials represent a powerful tool to investigate the magnetospheric plasma transport. The difficulty of deriving them for any magnetic configuration has generally reduced their use to that of the dipolar approximation. Our purpose is to compute the Euler potentials for non-dipolar magnetic configurations. Following Ho et al. (1997), we propose a simple numerical method which applies to regions of closed magnetic field lines. Section 2 briefly recalls some mathematical properties of the Euler potentials and Sect. 3 describes the numerical method used. It is then tested in Sect. 4 with the magnetic field model of Tsyganenko (1987).

\section{A short review of mathematical properties of Euler potentials}

The mathematical properties of these Euler potentials and their applications to physical problems have been reviewed in detail by Stern $(1970,1976)$. We will use two of their interesting mathematical properties: they are non unique and their tridimensional distribution can be derived from knowledge of how they map on a surface.

\subsection{Non uniqueness of Euler potentials}

Assume that one set of Euler potentials $\left(\alpha_{1}, \beta_{1}\right)$ has been derived for a particular magnetic field $\mathbf{B}$ :

$\mathbf{B}=\nabla \alpha_{1} \times \nabla \beta_{1}$

Consider two functions $\alpha_{2}\left(\alpha_{1}, \beta_{1}\right)$ and $\beta_{2}\left(\alpha_{1}, \beta_{1}\right)$. Then

$\nabla \alpha_{2} \times \nabla \beta_{2}=\left(\frac{\partial \alpha_{2}}{\partial \alpha_{1}} \frac{\partial \beta_{2}}{\partial \beta_{1}}-\frac{\partial \beta_{2}}{\partial \alpha_{1}} \frac{\partial \alpha_{2}}{\partial \beta_{1}}\right)\left(\nabla \alpha_{1} \times \nabla \beta_{1}\right)$

and $\alpha_{2}$ and $\beta_{2}$ will be a new set of Euler potentials provided that

$\left(\frac{\partial \alpha_{2}}{\partial \alpha_{1}} \frac{\partial \beta_{2}}{\partial \beta_{1}}-\frac{\partial \beta_{2}}{\partial \alpha_{1}} \frac{\partial \alpha_{2}}{\partial \beta_{1}}\right)=1$

Therefore, an infinite number of Euler potentials can be derived once one has been computed (Stern, 1970).

\subsection{Computation of Euler potentials from a surface}

If the topology of the magnetic field $\mathbf{B}$ is regular enough, it is not necessary to solve Eq. (2) in three dimensions due to the non-divergence of B (Stern, 1970, 1976).

We assume that Eq. (2) has been solved in the vicinity of one point $P$. We consider the field line $l_{P}$ passing through this point, and label it $\left(\alpha_{1}, \beta_{1}\right)$. As $\alpha_{1}$ and $\beta_{1}$ are constant along $l_{P}$ :

$\mathbf{B} \cdot \nabla \alpha_{1}=0$

$\mathbf{B} \cdot \nabla \beta_{1}=0$

which implies a function $F$ such that

$\mathbf{B}=F\left(\nabla \alpha_{1} \times \nabla \beta_{1}\right)$

exists. From Eq. (2), its value is unity at point $P$. Then, $\alpha_{1}$ and $\beta_{1}$, which are two independent functions, are completed by a third one, $\gamma_{1}$, to form a set of coordinates along $l_{P}$ generally not orthogonal. $\gamma_{1}$ can be, for instance, the linear distance along $l_{P}$ and allows us to label every point along $l_{P}$. With $F$ considered as a function of $\alpha_{1}, \beta_{1}$ and $\gamma_{1}$

$$
\nabla \cdot \mathbf{B}=\left(\frac{\partial F}{\partial \gamma_{1}}\right)\left(\nabla \gamma_{1}\right) \cdot\left(\nabla \alpha_{1} \times \nabla \beta_{1}\right)
$$

or

$$
\left(\frac{\partial F}{\partial \gamma_{1}}\right)\left(\nabla \gamma_{1}\right) \cdot\left(\nabla \alpha_{1} \times \nabla \beta_{1}\right)=0
$$

As $\alpha_{1}, \quad \beta_{1}$ and $\gamma_{1}$ are independent, the term $\left(\nabla \gamma_{1}\right) \cdot\left(\nabla \alpha_{1} \times \nabla \beta_{1}\right)$ does not cancel, and $\left(\frac{\partial F}{\partial \gamma_{1}}\right)$ vanishes. $F$ does not depend on $\gamma_{1}$ and is constant along $l_{P}$ where its value is unity as for point $P$. From Eq. (7)

$\mathbf{B}=\nabla \alpha_{1} \times \nabla \beta_{1}$

everywhere along $l_{P}$.

Therefore, it is sufficient to compute the Euler potentials at every point $P$ of a surface and the mapping along magnetic field lines will complete the 3D-distribution (Stern, 1970, 1976).

\section{Numerical computation method}

Stern (1970) described a general way to compute the Euler potentials on a surface in generalized orthogonal or non-orthogonal coordinates. We use this formalism to derive a first set of Euler potentials $\left(\alpha_{1}, \beta_{1}\right)$, and from it a second one $\left(\alpha_{2}, \beta_{2}\right)$ which is more convenient to use. We then describe the method to compute Euler potentials in regions of closed magnetic field lines and discuss its possible extension to open magnetic field lines.

\subsection{Derivation of a first set of Euler potentials}

One solves Eq. (2) on a plane surface $S$ crossed only once by the magnetic field lines. For instance, $S$ can be the equatorial plane of the magnetosphere or any other plane of physical interest. Such a plane avoids computing multiple-valued Euler potentials (Stern, 1970). 
Define a spherical $(r, \theta, \varphi)$ system relative to $S$, with $r$, $\theta$ and $\varphi$ respectively the radius, the colatitude and the azimuth of the point $P$ considered. The surface $S$ is defined by $\theta=\frac{\pi}{2}$. $\varphi=0$ corresponds to midnight, $\varphi=\pi$ to noon, $\varphi>0$ in the dawn sector and $\varphi<0$ in the dusk sector.

Equation (2) then gives a non linear system with 3 equations and 6 unknowns, the partial derivatives of $\alpha_{1}$ and $\beta_{1}$ with respect to $r, \theta$ and $\varphi$. It can be reduced to a 3-3 non-linear system if the expression of one Euler potential is given. For example, it can be convenient to choose $\alpha_{1}$ as a function of the volume $\vartheta$ of a flux tube of unit flux, $\alpha_{1}=\alpha_{1}(\vartheta)$ with

$\vartheta=\int \frac{\mathrm{d} s}{B}$

where $s$ is the distance along the closed magnetic field line and $B$ the module of $\mathbf{B}$. The integration is carried on between the two ionospheric ends of the field line. This particular choice is made in reference to Böstrom (1975), Peymirat and Fontaine (1994b) and Stern (1994b) who showed that the derivation of the magnetospheric plasma transport equations is simplified if $\alpha_{1}$ is function of $\vartheta$ only. Ho et al. (1997) chose the other point of view to compute the Euler potentials associated with Neptune's magnetic field. $\beta_{1}$ is imposed as a complex function numerically calculated, and $\alpha_{1}$ derived from $\beta_{1}$ with the relationship between the Euler potentials and the magnetic flux per unit area. We propose another general method to derive $\beta_{1}$.

The Euler potential $\beta_{1}$ is computed in $S$ from

$\mathrm{d} \beta_{1}=\left(\frac{\partial \beta_{1}}{\partial \mathrm{r}}\right) \mathrm{dr}+\left(\frac{\partial \beta_{1}}{\partial \varphi}\right) \mathrm{d} \varphi$

with $\theta=(\pi / 2)$ and hence $\mathrm{d} \theta=0$. Along a curve of constant value of $\alpha_{1}$ on $S$,

$\mathrm{d} \alpha_{1}=\left(\frac{\partial \alpha_{1}}{\partial \mathrm{r}}\right) \mathrm{dr}+\left(\frac{\partial \alpha_{1}}{\partial \varphi}\right) \mathrm{d} \varphi=0$

holds and Eq. (12) reduces to

$\mathrm{d} \beta_{1}=\frac{\left(\frac{\partial \alpha_{1}}{\partial \mathrm{r}} \frac{\partial \beta_{1}}{\partial \varphi}-\frac{\partial \beta_{1}}{\partial \mathrm{r}} \frac{\partial \alpha_{1}}{\partial \varphi}\right)}{\frac{\partial \alpha_{1}}{\partial \mathrm{r}}} \mathrm{d} \varphi$

The term in bracket is simplified from Eq. (2) projected on the $\theta$ axis

$\frac{\left(-\frac{\partial \alpha_{1}}{\partial r} \frac{\partial \beta_{1}}{\partial \varphi}+\frac{\partial \beta_{1}}{\partial r} \frac{\partial \alpha_{1}}{\partial \varphi}\right)}{r}=B_{\theta}$

where $B_{\theta}$ is the $\theta$ component of $\mathbf{B}$. Finally $\beta_{1}$ is obtained from

$\beta_{1}=\int_{0}^{\varphi} \mathrm{d} \beta_{1}=\int_{0}^{\varphi} \frac{-\mathrm{r} B_{\theta}}{\frac{\partial \alpha_{1}}{\partial \mathrm{r}}} \mathrm{d} \varphi$ or

$\beta_{1}=\int_{0}^{\varphi} \frac{-r B_{\theta}}{\frac{\partial \alpha_{1}}{\partial \vartheta} \frac{\partial \vartheta}{\partial r}} \mathrm{~d} \varphi$

where $\beta_{1}=0$ for $\varphi=0$ and the integration carried along a curve of constant $\alpha_{1}$. Equation (16) would be equivalent to the relationship used by Ho et al. (1997) if the unit area were the elementary area in the $(r, \theta, \varphi)$ space.

$\alpha_{1}(\vartheta)$ is defined similarly to the dipole approximation as

$\alpha_{1}(\vartheta)=-B_{0}^{0.75} R_{e}{ }^{1.25} \vartheta^{-0.25}$

where $B_{0}$ and $R_{e}$ are respectively the equatorial magnetic field on the Earth and the radius of the Earth.

\subsection{Derivation of a second set of Euler potentials with gauge constraints}

In the ideal case of the dipolar axisymmetrical magnetic field, symmetrical orthogonal Euler potentials $\alpha$ and $\beta$ can be derived (Stern, 1976). Symmetrical Euler potentials for symmetrical magnetic fields allow an easier interpretation of the physical properties of the studied system. Orthogonal Euler potentials, i.e. $\nabla \alpha \cdot \nabla \beta=0$, simplify the computations when they are used as coordinates. However, it is rarely possible to find orthogonal Euler potentials (Stern, 1994b) and we will limit our computation to Euler potentials complying with the symmetries of the magnetic field when they exist.

On the surface $S, \alpha_{1}$ defines a family of curves where $\alpha_{1}$ is constant. These curves cross the tail of the magnetosphere and encircle the Earth except at considerable distances in the noon sector where they are not defined due to the opening of the field lines. The third spherical coordinate $\varphi$ varies along these curves. We note $\varphi_{\max }\left(\alpha_{1}\right)$ its extremum value and $\beta_{1, \max }\left(\alpha_{1}\right)$ the corresponding value of $\beta_{1}$. In the case of a dipole, where the contours of $\alpha_{1}$ encircle the Earth, $\varphi_{\max }\left(\alpha_{1}\right)=\pi$ for every $\alpha_{1}$ and $\beta_{1, \max }\left(\alpha_{1}\right)=R_{e} \varphi_{\max }\left(\alpha_{1}\right)=R_{e} \pi$. In reference to the dipole, we take the following gauge constraint for the new set of Euler potentials $\left(\alpha_{2}, \beta_{2}\right)$

$\beta_{2}\left(\alpha_{1}, 0\right)=0$

$\beta_{2}\left(\alpha_{1}, \beta_{1, \max }\left(\alpha_{1}\right)\right)=R_{e} \varphi_{\max }\left(\alpha_{1}\right)$

with

$\alpha_{2}=\alpha_{2}\left(\alpha_{1}\right)$

In the case of a symmetrical magnetic field, Eqs. (19), (20) and (21) lead to symmetrical Euler potentials. If the magnetic field is not symmetrical, Euler potentials computed with Eqs. (19), (20) and (21) are not symmetrical but are such that $\beta_{2}=0$ at midnight, and $\beta_{2}=\pi R_{e}$ at noon whenever the contour of $\alpha_{2}$ is closed.

The integration of Eq. (4) along a curve of constant $\alpha_{1}$ with Eq. (21) leads to 


$$
\begin{aligned}
\beta_{2}\left(\alpha_{1}, \beta_{1}\right)= & \int_{0}^{\beta_{1}} \frac{\partial \beta_{2}}{\partial \beta_{1}^{\prime}} \mathrm{d} \beta_{1}^{\prime}+\beta_{2}\left(\alpha_{1}, 0\right) \\
& =\frac{1}{\frac{\partial \alpha_{2}}{\partial \alpha_{1}}} \beta_{1}+\beta_{2}\left(\alpha_{1}, 0\right)
\end{aligned}
$$

such that from Eqs. (19) and (20)

$$
\beta_{2}\left(\alpha_{1}, \beta_{1, \max }\left(\alpha_{1}\right)\right)=R_{e} \varphi_{\max }\left(\alpha_{1}\right)=\frac{1}{\frac{\partial \alpha_{2}}{\partial \alpha_{1}}} \beta_{1, \max }\left(\alpha_{1}\right)
$$

which implies

$\beta_{2}\left(\alpha_{1}, \beta_{1}\right)=\frac{R_{e} \varphi_{\max }\left(\alpha_{1}\right)}{\beta_{1, \max }\left(\alpha_{1}\right)} \beta_{1}$

from which one gets

$$
\begin{aligned}
\alpha_{2}\left(\alpha_{1}\right) & =\int_{\alpha_{1, \text { min }}}^{\alpha_{1}} \frac{\partial \alpha_{2}}{\partial \alpha_{1}^{\prime}} \mathrm{d} \alpha_{1}^{\prime}+\alpha_{2, \text { min }} \\
& =\int_{\alpha_{1, \text { min }}}^{\alpha_{1}} \frac{\beta_{1, \max }\left(\alpha_{1}^{\prime}\right)}{R_{e} \varphi_{\max }\left(\alpha_{1}^{\prime}\right)} \mathrm{d} \alpha_{1}^{\prime}+\alpha_{2, \text { min }}
\end{aligned}
$$

where $\alpha_{2, \min }=\alpha_{1, \min }$ is a constant given as in Eq. (18) as

$\alpha_{2, \min }=\alpha_{1}\left(\vartheta_{\min }\right)=-B_{0}^{0.75} R_{e}^{1.25} \vartheta_{\min }^{-0.25}$

with $\vartheta_{\min }$, the minimal value of $\vartheta$.

\subsection{Computation method for closed magnetic field lines}

The computation of the Euler potentials proceeds in two steps. One first calculates the volume $\vartheta$ from which $\alpha_{1}$ and $\beta_{1}$ are derived from Eqs. (17) and (18). Then, one calculates the second set of Euler potentials $\alpha_{2}$ and $\beta_{2}$ from Eqs. (24), (25) and (26). The knowledge of the magnetic field comes into play in Eqs. (17) and (18). The derivation of $\alpha_{1}$ implies a full tridimensional model to obtain the volume $\vartheta$, while the computation of $\beta_{1}$ makes only use of a bidimensional distribution of the $B_{\theta}$ component on a surface $S$. This method also applies similarly for two dimensional models of the magnetic field. In that case, the computation of the volume $\vartheta$ is not necessary. $\alpha_{1}$ is simply a given but single valued function that one arbitrarily chooses. $\beta_{1}$ is then derived from the same formula (17). In that way the method can directly be applied to a data set of magnetic field measurements. But the computation of the tridimensional distribution will then require field line tracing, which is possible if the quality of the data is good enough.

\subsection{Extension of the method to open magnetic field lines}

The method developed therebefore applies to regions of closed magnetic field lines. It can be extended to open magnetic field lines in the following way. Equation (2) is solved on a surface $S$ which is no more a plane but a sphere around the planet. The sphere must be sufficiently close to the planet such that any field line crosses the sphere at least once. Then one proceeds as in Sects. (3.1) and (3.2) and gets

$\beta_{1}=\int_{0}^{\varphi} \frac{r_{S}^{2} B_{r} \sin \theta}{\frac{\partial \alpha_{1}}{\partial \theta}} \mathrm{d} \varphi$

where $B_{r}$ is the $r$ component of $\mathbf{B}$, and $r_{S}$ the radius of the sphere.

The main difference and difficulty is the calculation of $\alpha_{1} . \alpha_{1}$ can be any single valued function except in regions of closed magnetic field lines where the surface $S$ is crossed twice because $\alpha_{1}$ must take the same value along the same field line. In regions of closed magnetic field lines, one must find on the surface $S$ the two conjugate hemispheres, which assumes that field line tracing is possible. On one hemisphere $\alpha_{1}$ can be any single valued function with the same value for the conjugate point of the other hemisphere. $\alpha_{1}$ must also be continuous and derivable along the equatorial boundary of the polar cap region of each hemisphere to avoid any non physical discontinuity.

\section{Application of the Euler potentials to the Tsyganenko model (Tsyganenko, 1987)}

The modelling of the magnetospheric field has developed considerably. The most recent models were proposed by Hilmer and Voigt (1995), Skone et al. (1995), Tsyganenko (1996), Ostapenko and Maltsev (1997), Tsyganenko (1997). The description of the magnetospheric currents differs from one model to the other. The input parameters are as different as the Dst index, the $K p$ index, the $A E$ index, the interplanetary magnetic field, the solar wind pressure, the magnetopause standoff distance, the midnight equatorward boundary of the diffuse aurora, the amount of stretching of the field lines and the orientation of the merging lines. Particular forms and versions (Donovan, 1993; Peredo et al., 1993; Tsyganenko, 1987, 1989, 1995) have been built to improve the consistency with the observations or for specific purposes. For example, Peredo et al. (1993) modified existing forms of the Tsyganenko (1987) and Tsyganenko (1989) models to better reproduce the observations in the near-tail magnetosphere; Lui et al. (1994) combined the models of Tsyganenko (1987) and Tsyganenko (1989) to model the quiet time nightside magnetosphere; Pulkkinen et al. (1994) modified the model of Tsyganenko (1989) to study the recovery phase of a substorm; Skone et al. (1995) improved the model of Donovan (1993) to compute the quiet time magnetic field at geostationary orbits; Kullen and Blomberg (1996) added the effect of the IMF in the model of Tsyganenko (1989). It is not our intention to describe in detail the numerous existing models, and the interested reader is referred, for example, to the review by Stern 
(1994b). We do not intend to test our general method to all the existing models of the magnetic field. For simplicity, we tested it on the model of Tsyganenko (1987) because it is simple, has been widely used in numerous developments, and contains the main features of the other models: a stretched tail, a dayside compression, open field lines at high latitudes and a dependence on the magnetic activity.

The short version of the external field valid up to 30 $R_{e}$, which is more accurate than the long version (Tsyganenko, 1987), is considered. The main field is the dipole field and the geodipole tilt angle is set to zero. The computations are performed between $2 R_{e}$ where the field is very dipolar, and the region where the field lines open $\left(r<30 R_{e}\right)$. We use a spatial grid in the equatorial plane of the magnetosphere, with a step of $0.01 R_{e}$ for radial distances $r$ and $(2 \pi / 80)$ for azimuthal angles $\varphi$. The small radial step is chosen in order to compute with a good accuracy the radial derivatives of the unit flux tube volume $\vartheta$ (Eq. 17).

Figure 1a, b illustrates in the GSM coordinates the field lines of the Tsyganenko (1987) model in the noonmidnight meridian plane for $3-<K p<3+$ (top) and $5-<K p$ (bottom) corresponding respectively to moderate and disturbed magnetic activity. The bold lines at polar latitudes are connected to the poles; at lower latitudes, they represent the external boundary of the domain, i.e. the last closed field lines that we consider to compute the Euler potentials. The field lines become a little more compressed in the noon sector and more stretched in the midnight sector with the magnetic activity.

Figure 1c, d shows the equatorial conjugates (ZGSM $=0$ ) of points of the ionosphere with invariant latitudes between $0^{\circ}$ and $90^{\circ}$ with a $0.1^{\circ}$ step for two geomagnetic activity levels $(3-<K p<3+; 5-<K p)$. Close to the
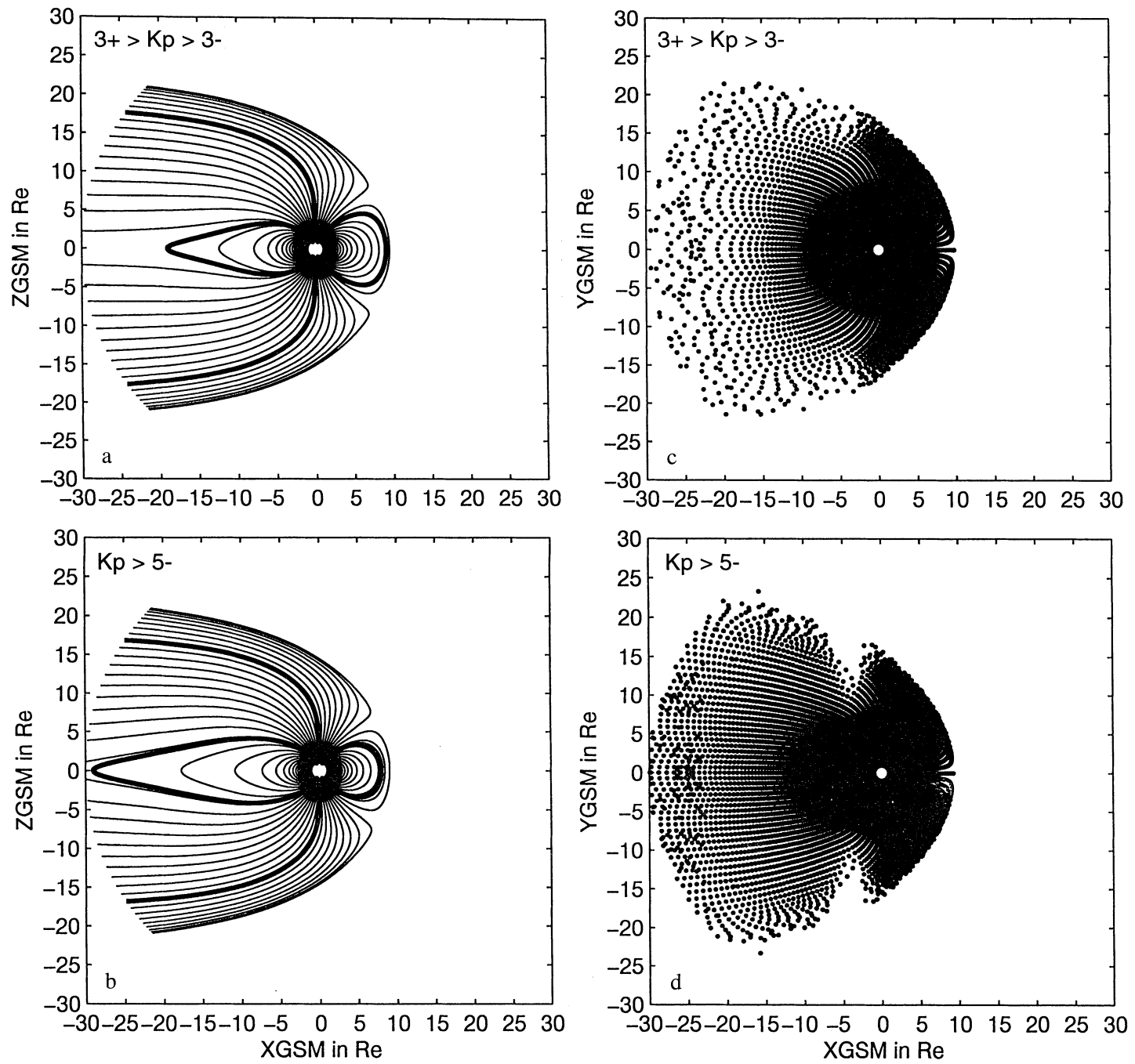

Fig. 1a-d. Field lines in the XGSM-ZGSM plane of the Tsyganenko (1987) magnetic field model every $2^{\circ}$ invariant magnetic latitude from the North Pole with the dipole field as the main field and zero geodipole tilt angle. The bold lines correspond to the poles at high latitudes and to the external boundary of the considered domain at low latitudes. a Corresponds to $3-<K p<3+$ where the external boundary is set to $19 R_{e}$ in the nightside and $9.13 R_{e}$ in the noon side, and $\mathbf{b}$ to $5-<K p$ where it is set to $29 R_{e}$ in the nightside and $8.03 R_{e}$ in the dayside $\mathbf{c}, \mathbf{d}$ Projection of the ionospheric invariant latitudes on the equatorial plane of the magnetosphere from $90^{\circ}$ to $0^{\circ}$ every $0.1^{\circ}$ in the XGSM-YGSM coordinates. c Corresponds to $3-<K p<3+$ and $\mathbf{d}$ to $5-<K p$ 
Earth, the field lines are closed and map to the equatorial plane of the magnetosphere in a regular and continuous way such that the equatorial plane is full of points. Far away from the Earth, the field lines open, corresponding in our definition to radial distances larger than $30 R_{e}$, and the equatorial plane starts to empty. An interesting feature is the fish shape of Fig. 1d, showing the influence of the magnetic activity on the field lines close to the polar cap.

We use the equatorial plane of the magnetosphere to compute the Euler potentials. The results are first presented as equivalent McIlwain parameters, then in the equatorial plane of the magnetosphere and finally in the ionosphere.

\subsection{Equivalent L McIlwain parameter}

The L McIlwain parameter corresponds approximately to the largest geocentric distance, expressed in earth radii, attained by a field line (Stern, 1976). In the case of the dipole, $L$ is obtained in the equatorial plane of the magnetosphere where the following relationship holds between $L$ and $\alpha$

$L(\alpha)=\frac{-B_{0} R_{e}}{\alpha}$

As suggested by Stern (1976), we generalize this relation to a non dipolar magnetic field. This parameter, called equivalent McIlwain parameter, allows an interpretation from a comparison to the dipole.

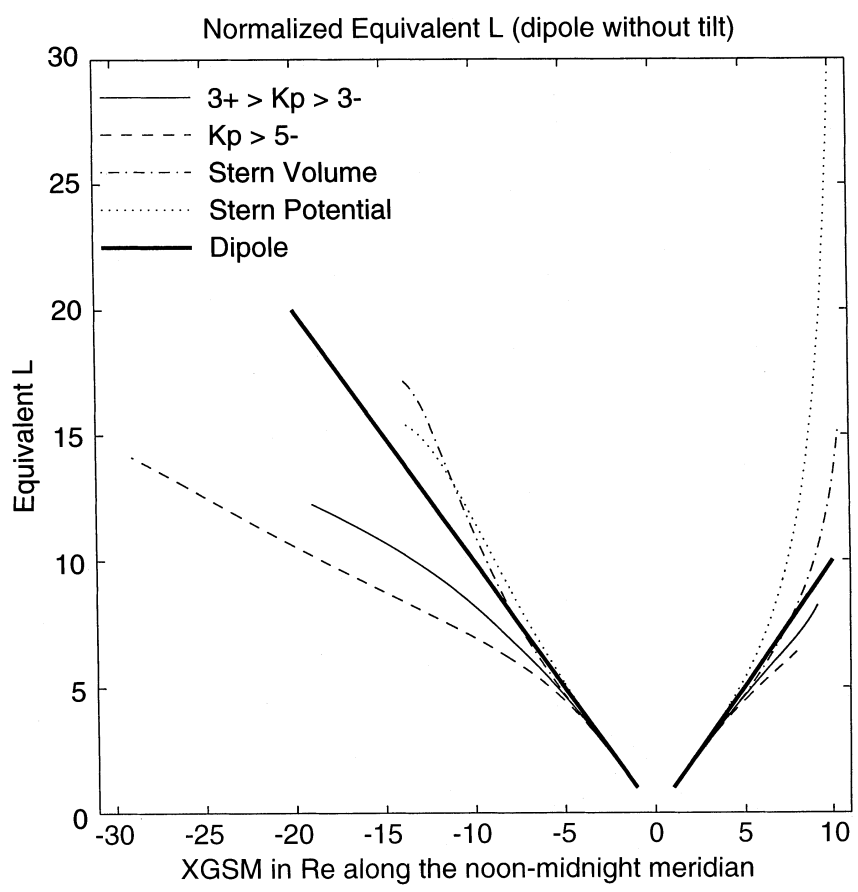

Fig. 2. Normalized equivalent $L$ parameter as a function of XGSM in the noon-midnight meridian plane. The plain line corresponds to $3-<K p<3+$, the dashed line to $5-<K p$, the dotted-dashed line to $L$ computed from the volume $\vartheta$ of the Stern model, the dotted line to $L$ inferred by Stern (1967) and the bold line to the dipole
Figure 2 shows $L$ as a function of XGSM in the noon-midnight meridian plane for the magnetic field models of Stern (1967) and of Tsyganenko (1987). The dotted and dotted-dashed lines correspond to the magnetic field model built by Stern (1967) and completely described by analytical Euler potentials. He used a perturbation method to derive them from the magnetic field model of Mead (1964). The dotted line illustrates $L$ given by Stern (1967), the dotted-dashed line the normalized $L$ that we computed from Eqs. (2426) and the volume $\vartheta$ associated to his magnetic field model, and the bold line the dipole. Indeed, the computation of the label $L$ of magnetic field lines depends on the various choices made for the Euler potentials, and which may have no relationship between them. We note that close to the Earth, below 5 $\mathrm{Re}$, the curves are identical and coincide with the dipole. When the distance increases, the two curves depart from the dipole $L$ becoming larger than the dipole one. The two curves behave approximately similarly in the midnight sector, but differ more significantly in the dayside near the magnetopause. As they represent the same magnetic field model, this difference illustrates the only effect on mapping on different Euler potentials. In general, it is not the signature of physical processes, such as the compression or extension of magnetic field lines.

The plain and dashed lines of Fig. 2 illustrate the normalized $L$ parameter that we computed for the Tsyganenko model from Eqs. (24-26) for moderate and disturbed magnetic conditions. For both activity levels, in the noon and midnight sectors, the equivalent $L$ is found to be smaller than the dipole and than the previous curves computed from Stern's model (Stern, 1967). Again, this result cannot be directly related to any physical processes. However, one particular feature allows us to go a little further in the comparison of results for both activity levels from Tsyganenko's model (Tsyganenko, 1987): the equivalent parameter $L$ maps down to the same latitude in the ionosphere. This means that the ionospheric footprint of a given field line labelled by a given equivalent $L$ does not depend on the activity. The main effect of the activity can be seen in Fig. 2 in the nightside: it increases the geocentric distance of the field line in the equatorial plane, which can be interpreted this time as the signature of the tail-like extension with activity. In the dayside, the curves are very close, which tends to indicate that the activity level has only a weak influence on the dayside magnetic field, as illustrated in Fig. 1a, b.

\subsection{Equatorial distribution of the Euler potentials}

The distributions of the Euler potentials associated with the Tsyganenko model are illustrated in the equatorial plane of the magnetosphere in Fig. 3a, b for moderate (top) and disturbed magnetic activity (bottom). For $5-<K p$, the shape of the domain where the Euler potentials are computed looks like a spear head to avoid the opening of the field lines in the 0400-0600 MLT and the 1800-2000 MLT sectors illustrated in Fig. 1d. 

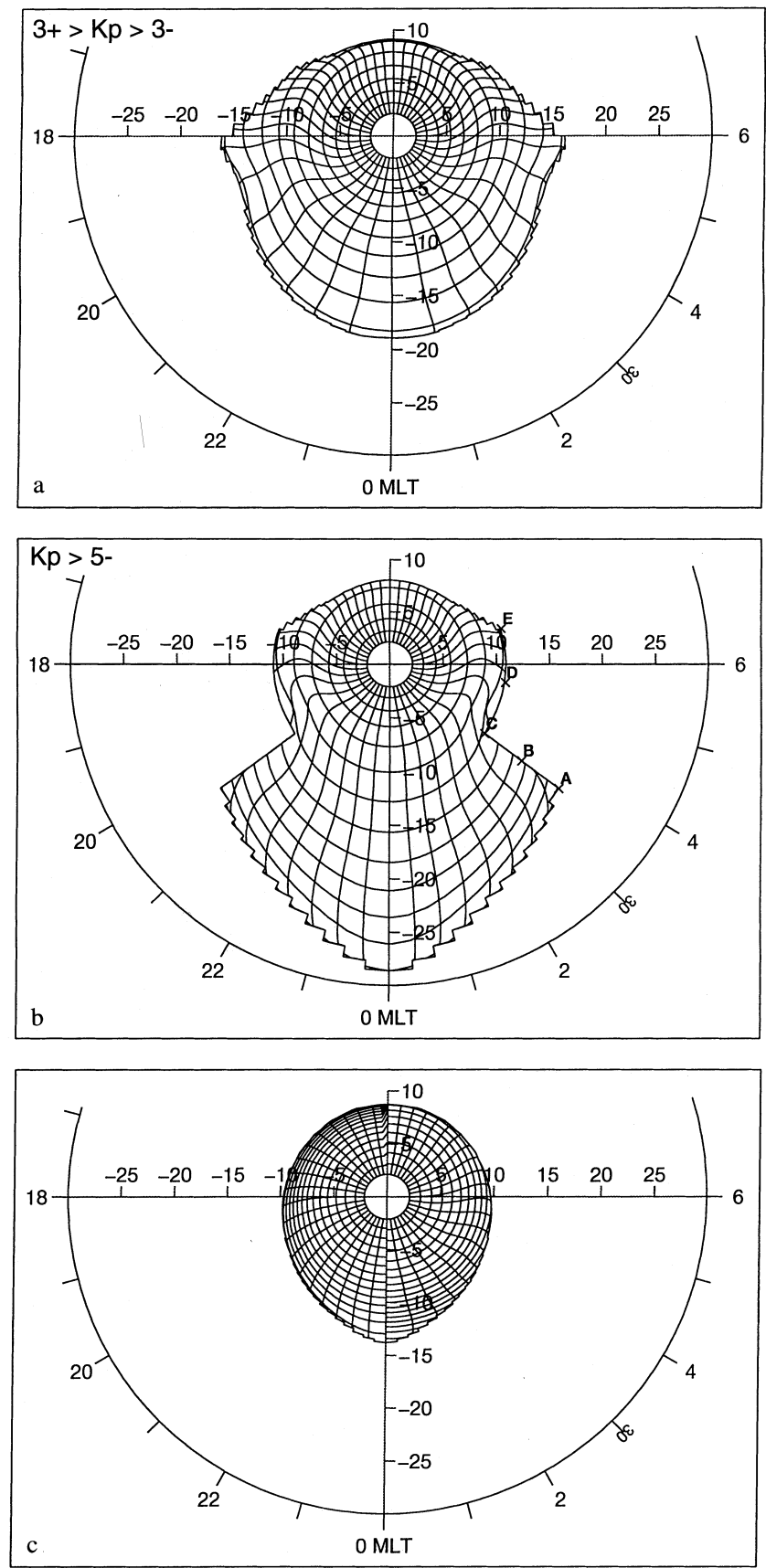

Fig. 3a-c. Contours of the Euler potentials in the equatorial plane of the magnetosphere between the Earth's centre and $30 R_{e}$ with the Magnetic Local Time indicated on the external circle a for $3-<K p<3+$ and $\mathbf{b}$ for $5-<K p$. The $\alpha$ contours are represented as contours of the equivalent $L$ parameter. They encircle the Earth while the $\beta$ contours cross them. The contour interval is 1 for $L$ with the closest contour to the Earth corresponding to 2 , and is $\left(2 \pi R_{e} / 40\right)$ for $\beta$ with $\beta=0$ in the midnight meridian $\mathbf{c}$ Contours of the Euler potentials in a format similar to $\mathbf{a}, \mathbf{b}$. The dusk sector $(1200<$ MLT $<2400)$ corresponds to the Euler potential inferred by Stern (1967). The dawn sector illustrates the Euler potentials that we compute from the volume $\vartheta$ of the Stern (1967) magnetic field model

In Fig. 3a, b, the contours of $\alpha_{2}$ are represented by the corresponding contours of equivalent $L$, from $L=2$ and steps of one unit. Close to the Earth, they are circles around the Earth as for a dipole. The only difference is a $L$-value smaller than the radial distance: for example, for disturbed magnetic activity, the contour $L=6$ is located approximately at $r=8 R_{e}$ at 0000 MLT. The contours become non-concentric when the distance increases in the midnight sector. Antonova and Ganushkina $(1996,1997)$ computed the volumes associated with the Tsyganenko model for a lower magnetic activity, $K p=2$, and obtained the same behaviour. When the magnetic activity increases, the contours become more stretched at larger distances in the tail in the nightside. Stern (1967) used the magnetospheric model of Mead (1964), and Cheng (1995) solved the Grad-Shafranov equation with a given profile of the magnetospheric pressure to compute the Euler potentials. They obtained similar results except at noon where their contours approach closer to the Earth with the magnetic activity. This is due to the normalization used in the computation of the Euler potentials (Eqs. 24-26) as discussed in Sect. 4.1.

The contours of $\beta_{2}$ are represented by the radial curves crossing the contours of $L$. When the radial distance increases, they first rotate towards the dayside, then in the opposite direction towards the nightside. The bending of the contours of $\beta_{2}$ increases with the magnetic activity illustrating the effect of the solar wind on the magnetic field and the departure from a dipole where the contours of $\beta_{2}$ are the radial lines $\varphi=$ constant. Similar contours are computed by Stern (1967) with the magnetic field model of Mead (1964) but where the only rotation is towards the nightside. The rotation towards the dayside in the Tsyganenko model is not due to the normalization as we checked it with the non normalized Euler potentials $\alpha_{1}$ and $\beta_{1}$. As $\beta_{1}$ is computed along the $\alpha_{1}$ contours from $B_{\theta}$, it depends in a complex way on the magnetic field model and we did not find any simple explanation. We shall return to it in the next paragraph.

Figure 3c shows the normalized Euler potentials $\alpha_{2}$ and $\beta_{2}$ associated with the Stern model (1967), with the Euler potentials calculated by Stern between 1200 and 2400 MLT, and the Euler potentials that we compute between 0000 MLT and 1200 MLT from the volume $\vartheta$ of the Stern model (1967). The difference between both potentials clearly shows that several sets of Euler potentials can be found for a given magnetic field depending on the computation method. Close to the Earth where the magnetic field is nearly dipolar, the $L$ contours that we computed are slightly displaced tailwards in comparison to the dipole where they are located at a distance $L$. This is due to Eq. (18) which is an estimate of the true formula for the dipole where the quadratic terms have been neglected for simplicity. Interestingly, the $\beta$ contours are slightly rotated toward the dayside close to the Earth as for the Tsyganenko model. This rotation is not obtained by Stern, and depends on the choice of $\alpha$.

Figure 4 illustrates in the equatorial plane of the magnetosphere for moderate magnetic activity, the contours of the north-south component $B_{\theta}$ computed (Fig. 4a) directly from the model of Tsyganenko (1987) and (Fig. 4b) from the Euler potentials that we just 
derived and displayed in the previous paragraph (see Fig. 3a). Both results coincide over the restricted domain where we computed the Euler potentials, which shows the precision of our computation method although Euler potentials are calculated with a precision of about $1.5 \%$. Similar agreements are obtained for the case of large magnetic activity.

\subsection{Ionospheric projection of the Euler potentials}

Figure 5a, b represents polar maps of the ionosphere with the pole at the centre and latitudes above $40^{\circ}$. They display the ionospheric projection of the contours of $L$ and $\beta_{2}$ along the magnetic field lines for moderate (Fig. 5a) and great (Fig. 5b) magnetic activity. At lower latitudes, the contours of $L$ are very similar to those of a dipole. At higher latitudes, they concentrate on the nightside due to the field lines stretching in the nightside and the small compression of the field lines in the dayside. Antonova and Ganyushkina (1995 and references therein) and Peymirat and Fontaine (1994a)
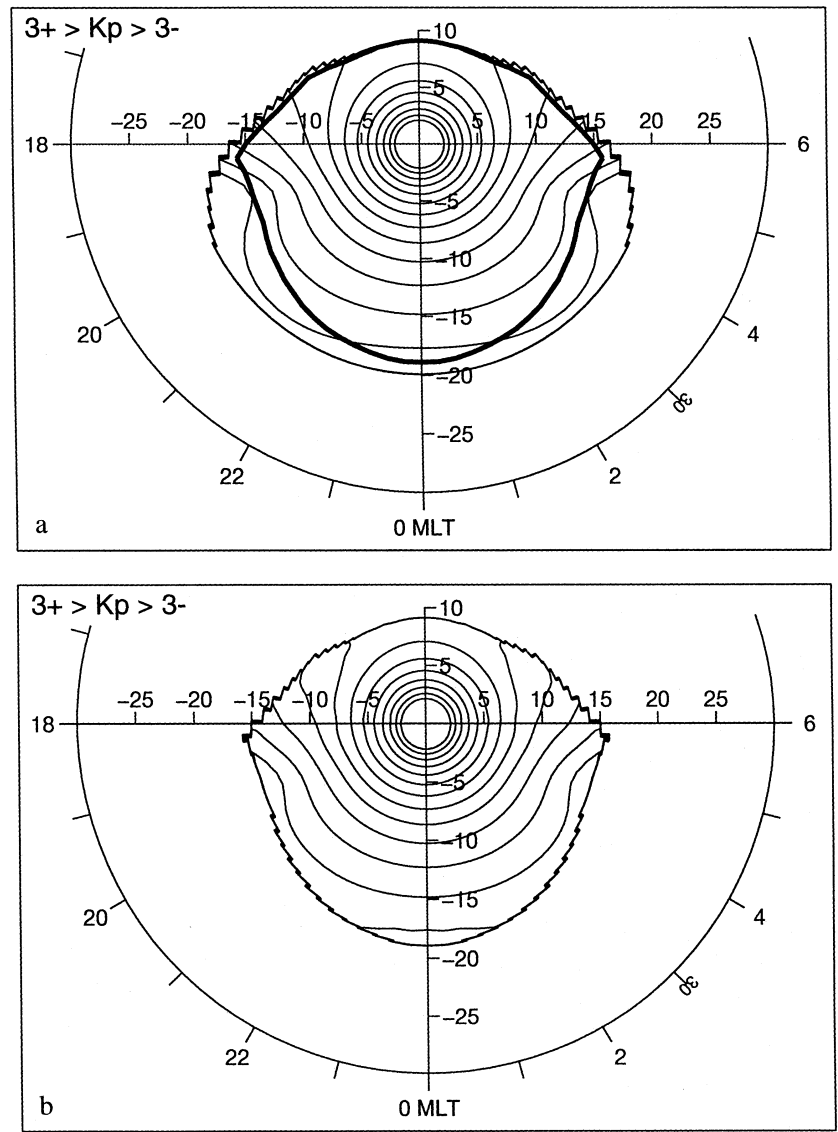

Fig. 4a, b. Contours of the $B_{\theta}$ component (in nT) in equatorial maps similar to Fig. $3 \mathrm{a}, \mathrm{b}$ for $3-<K p<3+$. The $B_{\theta}$ contours are represented as contours of the quantity $\log \left(B_{\theta}\right)$. The contour interval is 0.25 and the closest contour to the Earth corresponds to 3.5. $B_{\theta}$ is given from the Tsyganenko (1987) model in a and computed from the Euler potentials illustrated in Fig. $3 \mathrm{a}$ in $\mathbf{b}$. The bold line in a corresponds to the external boundary of the domain where the Euler potentials are computed
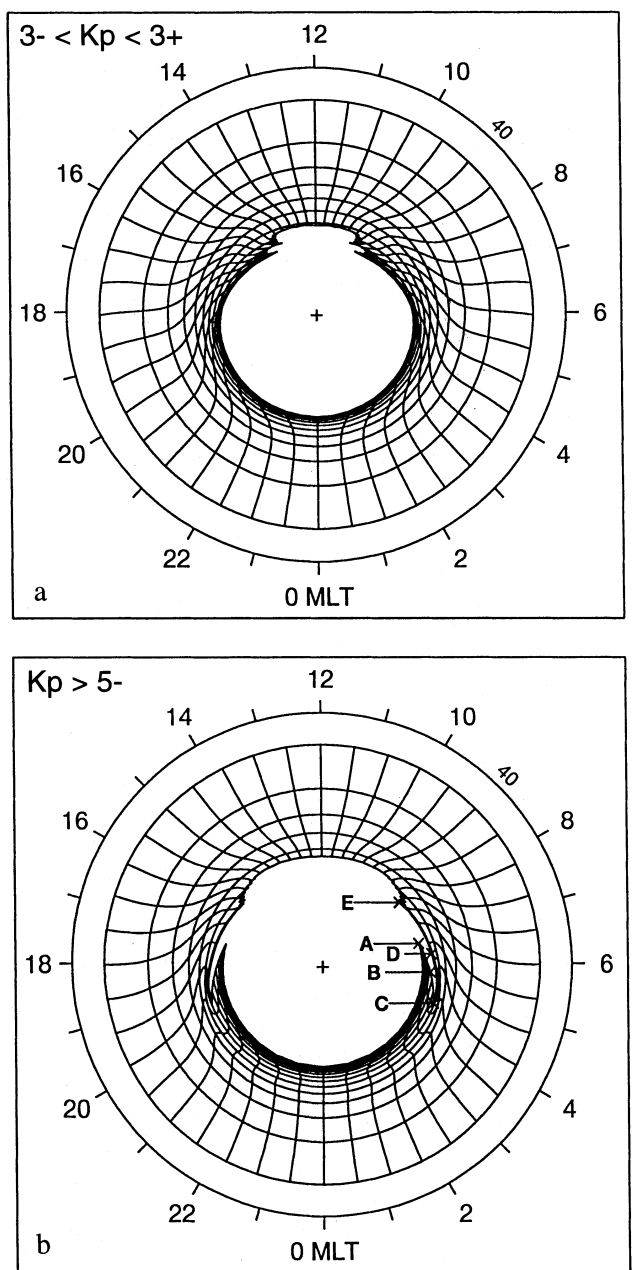

Fig. 5a, b. Ionospheric map of the Euler potentials between the North Pole and $40^{\circ}$ invariant latitude with the Magnetic Local Time indicated on the external circle a for $3-<K p<3+$ and $\mathbf{b}$ for $5-<K p$. The contour format is similar to Fig. 3a, b. The points $A, B, C, D$ and $E$ are mapped down to the equatorial plane of the magnetosphere and their equatorial conjugates are indicated in Fig. $3 b$

computed for various magnetic activities the volume of unit magnetic flux tubes using the magnetic field of Tsyganenko (1987) but with the Internal Geophysical Reference Field $(1985,1986)$ as the main field. Their results are in agreement with ours.

The contours of $\beta_{2}$ start as the dipole for low latitudes and become distorted at higher latitudes. The distortion increases again with the magnetic activity. Wings appear in the mapping at dawn and dusk at the polar cap boundary for large magnetic activity. They reflect the particular domain that we used in the equatorial plane of the magnetosphere to avoid the regions with strongly distorted field lines close to the polar cap as illustrated by the points A, B, C, D and E. Their equatorial conjugates are indicated in Fig. 3b. The mapping is regular except for points A and B which map at the same MLT in the equatorial plane of the magnetosphere. The field lines connecting these two points to their equatorial conjugates originate from the dayside of the ionosphere and bend towards the tail due to the solar wind. 


\section{Conclusion}

The Euler potentials $(\alpha, \beta)$ provide a very useful set of coordinates for the description of the magnetic topology. They simplify the derivation of the basic equations of the plasma transport in the magnetosphere and the mapping for studies related to the coupling between the ionosphere and the magnetosphere.

We proposed a numerical method to compute the Euler potentials based on a formalism previously made by Stern (1970) and which applies to regions of closed field lines. The Euler potentials are first computed on a surface, and their 3-D distribution is then obtained by magnetic field line mapping. We chose for this initial surface the equatorial plane of the magnetosphere, which is the simplest surface crossed only once by closed magnetic field lines. The results are represented both in the equatorial plane, and in the ionosphere.

The pair $(\alpha, \beta)$ of Euler potentials describing a magnetic field is not unique. Ho et al. (1997) assumed that $\beta$ is given as a complex function numerically computed from which they derived $\alpha$ using the relationship between the Euler potentials and the magnetic flux per unit area. We propose another general method that reduces to the one used by Ho et al. (1997) if the unit area considered is the elementary area in the spherical coordinates space. It assumes that $\alpha$ only depends on the volume $\vartheta$ of a flux tube of unit magnetic flux with the constraint that $\beta=0$ at midnight and that the existing symmetries of the magnetic field are preserved. The method is developed to recover the usual pair of Euler potentials in the case of a dipolar magnetic field. In fact, the conditions mentioned are not absolutely necessary and the method developed here is much more general. For example, it can be directly applied to a set of magnetic field measurements if the dependence of $\alpha$ on $\vartheta$ is disregarded, or extended to open magnetic field lines if the considered surface is a sphere around the planet.

The method is illustrated with the model of Tsyganenko (1987). In the magnetosphere, close to the Earth, the Euler potentials are similar to those of a dipole, the departure from the dipole increasing with the distance and the magnetic activity. The $\alpha$ contours are illustrated as contours of the equivalent McIlwain parameter $L$ (Stern, 1976). In the nightside, the main effect of the magnetic activity is to stretch tailward the magnetic field lines. On the dayside, it has practically no influence. The contours of $\beta$ are rotated towards the dayside close to the Earth, and are also stretched with the magnetic activity in the nightside. The dayside rotation is associated to the choice of $\alpha$. Finally, the Euler potentials are mapped on the ionosphere along the magnetic field. The departure from a dipole increases with the latitude and the magnetic activity. At large latitudes, the contours of $\beta$ display a distortion increasing with the magnetic activity. The numerical method illustrated with the model of Tsyganenko (1987) can be applied to a wide class of magnetic fields in regions of closed field lines for studies of the magnetospheric convection coupled to the ionosphere. In particular, we intend to implement it in our numerical model of the magnetospheric transport (Peymirat and Fontaine, 1994b) to derive the physical consequences of a magnetic field distribution more realistic than the dipolar approximation.

Acknowledgements. Topical Editor K.-H. Glassmeier thanks O. Amm and another referee for their help in evaluating this paper.

\section{References}

Antonova, E. E., and N. Y. Ganyushkina, On deciding on the coordinate system for describing magnetostatically equilibrated regions of the magnetosphere, Geomagn. Aeron., 34, 479-486, 1995.

Antonova, E. E., and N. Y. Ganushkina, Azimuthal hot plasma pressure gradients and dawn-dusk electric field formation, Preprint 96-4/411, Skobeltsyn Institute of Nuclear Physics Moscow State University, Moscow 1996.

Antonova, E. E., and N. Y. Ganushkina, Azimuthal hot plasma pressure gradients and dawn-dusk electric field formation, J. Atmos. Solar-Terr. Phys., 59, 1343-1354, 1997.

Boström, R., Mechanisms for driving Birkeland currents, in Physics of the Hot Plasma in the Magnetosphere, Ed. B. Hultqvist., Plenum Press, N. Y., 314-365, 1975.

Cheng, C. Z., Three-dimensional magnetospheric equilibrium with isotropic pressure, Geophys. Res. Lett., 22, 2401-2404, 1995.

Donovan, E. F., Modeling the Magnetic effects of field-aligned currents, J. Geophys. Res., 98, 13 529-13 543, 1993.

Hilmer, R. V., and G. H. Voigt, A magnetospheric magnetic field model with flexible current systems driven by independent physical parameters, J. Geophys. Res., 100, 5613-5626, 1995.

Ho, C. W., T. S. Huang, and S. Gao, Contributions of the highdegree multipoles of Neptune's magnetic field: an Euler potentials approach, J. Geophys. Res, 102, 24 393-24 401, 1997.

Khurana, K. K., Euler potential models of Jupiter's magnetospheric field, J. Geophys. Res., 102, 11 295-11 306, 1997.

Kullen, A., and L. G. Blomberg, The influence of IMF By on the mapping between the Earth's magnetotail and its ionosphere, Geophys. Res. Lett., 18, 2561-2564, 1996.

Lui, A. T. Y., H. E. Spence, and D. P. Stern, Empirical modeling of the quiet time nightside magnetosphere, J. Geophys. Res., 99, 151-157, 1994.

Mead, G. D., Deformation of the geomagnetic field by the solar wind, J. Geophys. Res., 69, 1181-1195, 1964.

Ostapenko, A. A., and Y. P. Maltsev, Relation of the magnetic field in the magnetosphere to the geomagnetic and solar wind activity, J. Geophys. Res., 102, 17 467-17 473, 1997.

Peredo M., D. P. Stern, and N. A. Tsyganenko, Are existing magnetospheric models excessively stretched, J. Geophys. Res., 98, 15 343-15 354, 1993.

Peymirat, C., and D. Fontaine, Relationships between field-aligned currents and convection observed by EISCAT and implications concerning the mechanism that produces region-2 currents: statistical study, Ann. Geophysicae, 12, 304-315, 1994a.

Peymirat, C., and D. Fontaine, Numerical simulation of magnetospheric convection including the effect of field-aligned currents and electron precipitation, J. Geophys. Res., 99, 11 155-11 176, 1994b.

Pulkkinen, T. I., D. N. Baker, P. K. Toivanen, R. J. Pellinen, R. H. W. Friedel, and A. Korth, Magnetospheric field and current distributions during the substorm recovery phase, J. Geophys. Res., 99, 10 955-10 966, 1994.

Skone, S. H., E. F. Donovan, and G. Rostoker, Characterizing the quiet time magnetic field at geostationary orbit, J. Geophys. Res., 100, 23 583-23 596, 1995. 
C. Peymirat, D. Fontaine: A numerical method to compute Euler potentials for non dipolar magnetic fields

Stern, D. P., Geomagnetic Euler potentials, J. Geophys. Res., 72, 3995-4005, 1967.

Stern, D. P., Euler potentials, Am. J. Phys., 38, 494-501, 1970.

Stern, D. P., Representation of magnetic fields in Space, Rev. Geophys., 14, 199-214, 1976.

Stern, D. P., Tail modeling in a stretched magnetosphere, 1., methods and transformations, J. Geophys. Res., 92, 4437-4448, 1987.

Stern, D. P., Euler potentials of current-free fields expressed in spherical harmonics, J. Geophys. Res., 99, 2443-2445, 1994a.

Stern, D. P., The art of mapping the magnetosphere, J. Geophys. Res., 99, 17 169-17 198, 1994b.

Tsyganenko, N. A., Global quantitative models of the geomagnetic field in the cislunar magnetosphere for different disturbance levels, Planet. Space Sci., 35, 1347-1358, 1987.
Tsyganenko, N. A., A magnetospheric magnetic field model with a warped tail current sheet, Planet. Space Sci., 37, 15-20, 1989.

Tsyganenko, N. A., Modeling the Earth's magnetospheric magnetic field confined within a realistic magnetopause, J. Geophys. Res., 100, 5599-5612, 1995.

Tsyganenko, N. A., Effects of the solar wind conditions on the global magnetospheric configurations as deduced from data-based field models, in Proceedings of the ICS-3 Conference, Versailles, France, May 12-17, 1996, ESA SP-389, pp. 181-185, 1996.

Tsyganenko N. A., An empirical model of the substorm current wedge, J. Geophys. Res., 102, 19 935-19 941, 1997.

Tsyganenko, N. A., and D. P. Stern, Modeling the global magnetic field of the large-scale Birkeland current systems, J. Geophys. Res., 101, 27 187-27 198, 1996. 